\title{
Los juegos como estrategia metodológica en la enseñanza de la geometría, en estudiantes de séptimo grado de educación básica
}

\begin{tabular}{ccc} 
Carmelo Bravo. & Henry Márquez. & Felicia Villarroel. \\
\hline bravocarmelo@hotmail.com & henrylmarquez@gmail.com & feliciavillarroel@gmail.com \\
Departamento de Matemáticas & Departamento de Matemáticas & Departamento de Matemáticas \\
Universidad de Oriente, Venezuela & Universidad de Oriente, Venezuela & Universidad de Oriente, Venezuela
\end{tabular}

Resumen. El objetivo principal de esta investigación es el de presentar los juegos como estrategia metodológica en la enseñanza de la geometría, con el propósito de mejorar el rendimiento escolar de la geometría en séptimo grado de Educación Básica en la U.E.L.B “Ricardo Márquez Moreno”, ubicada en Santa Ana, estado Nueva Esparta, República Bolivariana de Venezuela, durante el año escolar 2008-2009. El presente estudio se enmarca en la modalidad de investigación de campo de tipo descriptivo. La población la conformaron doscientos (200) estudiantes y ocho (08) docentes del área de matemáticas. La muestra estuvo representada por 50 estudiantes integrantes de dos (2) secciones. Los instrumentos utilizados para recabar la información fueron dos cuestionarios, uno aplicado a los docentes y el otro a los estudiantes. El análisis de los resultados indicó que los docentes utilizan estrategias tradicionales para la enseñanza de la geometría como, por ejemplo, la exposición y muy pocas veces ponen en práctica la estrategia de los juegos. Además se determinó que los estudiantes necesitan motivación e integración hacia la geometría mediante estrategias motivadoras y agradables como los juegos didácticos, por lo cual se sugiere el uso de estas estrategias para mejorar el rendimiento y la calidad educativa.

Palabras clave: Juegos, Estrategias Metodológicas, Enseñanza, Aprendizaje, Geometría.

Abstract. The main objective of this investigation is of presenting the games as methodological strategy in the education of the geometry, with the intention of improving the school performance of the geometry in the seventh degree of Basic Education in the U.E.L.B "Ricardo Márquez Moreno", located in Santa Ana, Estado Nueva Esparta, Republica Bolivariana de Venezuela, during the school year 2008-2009. The present study places in the modality of field investigation of descriptive type. The population was shaped by two hundred (200) students and eight (08) teachers of the area of mathematics. The sample was represented by 50 integral students of two (2) sections. The instruments used to obtain the information were two questionnaires, one applied to the teachers and other one to the students. The analysis of the results indicated that the teachers use traditional strategies for the education of the geometry as, for example, the exhibition and very rarely they put into practice the strategy of the games. In addition one determined that the students need motivation and integration towards the geometry by means of motivating and agreeable strategies as the didactic games, by which is suggested the use of these strategies to improve the performance and the educational quality.

KeyWords: Games, Methodological Strategies, Teaching, Learning, Geometry.

\subsection{Introducción}

Zambrano (2005) sostiene que la didáctica de la matemática "es la disciplina científica cuyo objeto es la génesis, circulación y apropiación del saber matemático y sus condiciones de enseñanza y aprendizaje" (p.5). Por ello 
es necesario que los docentes de matemáticas, asimilen la importancia de la didáctica de esta disciplina, a fin de buscar alternativas metodológicas para que los estudiantes constructores de su propio aprendizaje se apropien de esos saberes matemáticos.

Particularmente en el área de geometría, los docentes de Educación Básica tienen la tarea de resaltar las grandes virtudes y fortaleza que ella ofrece, al brindar oportunidades a los estudiantes para que se ubiquen en el espacio que los rodea, de tal manera que puedan observar, reconocer y describir las formas de las figuras de su entorno inmediato $y$, en consecuencia establecer relaciones entre espacio y forma.

Para ello, se requiere de un docente mediador del aprendizaje, que propicia ambientes adecuados con base en la utilización de materiales concretos provenientes de su propio entorno, para estimular el interés y la creatividad de los estudiantes, y el gusto y placer por aprender matemáticas.

La problemática observada en la enseñanza de la matemática está determinada por los enfoques metodológicos que aplican los docentes en ese campo; es necesario revisar los métodos y recursos que se utilizan, sobre todo en los niveles de Educación Básica. Dentro de estos recursos surge el juego como estrategia para la enseñanza de la matemática.

La estrategia de los juegos cobra gran importancia en el estudio de la geometría, sobre todo en los juegos de papel y lápiz, los cuales facilitan el trazado de figuras y líneas geométricas, agiliza los procesos mentales, agudiza el razonamiento lógico, entre otras cosas.

De lo anterior se desprende la importancia de implementar estrategias novedosas que desarrollen destrezas potenciales y habilidades en el adolescente que le lleven a construir progresivamente el conocimiento geométrico, constituyendo los juegos pedagógicos una estrategia para la enseñanza exitosa para el área de la geometría y además de propiciar la oportunidad de tener docentes abiertos al cambio, que mediante estos juegos logren inducir a los estudiantes en el aprendizaje de la geometría.

El objetivo principal de esta investigación es el de presentar los juegos como estrategia metodológica en la enseñanza de la geometría, que permitan mejorar el rendimiento escolar de la geometría en séptimo grado de Educación Básica en la U.E.L.B “Ricardo Márquez Moreno”, de Santa Ana, estado Nueva Esparta, República Bolivariana de Venezuela.

\subsection{Planteamiento de la investigación}

En un estudio realizado en todo el país por el Sistema Nacional de Medición y Educación del Aprendizaje (SINEA 1998), para evaluar las competencias que poseían los discentes de las tres etapas, de la entonces, Educación Básica, se determinó que en el área de matemáticas los alumnos no lograban alcanzar los niveles de ejecución requeridos. En este sentido se evaluaron los tópicos de: números y operaciones, geometría, organización y representación de datos e informática. En relación a la geometría, los educandos demostraron deficiencia en cuanto al dominio de las relaciones espaciales y su expresión en términos matemáticos.

De acuerdo al Centro Nacional para el Mejoramiento de la Enseñanza de la Ciencia (CENAMEC, 2001), en el Encuentro "Compartiendo Experiencias en las Áreas de Matemáticas y Ciencias de la Naturaleza y Tecnología", en la enseñanza de la geometría ha predominado el carácter formal, basado en la enseñanza de la geometría, en el lenguaje de álgebra vectorial y deductivo para el nivel de básica, fomentando, de esta manera, el aprendizaje memorístico que resulta contraproducente en la formación de adolescentes.

Los juegos como estrategia metodológica . Carmelo Bravo et al.

Derechos Reservados @ 2012 Revista digital Matemática, Educación e Internet (www.tec-digital.itcr.ac.cr/revistamatematica/) 
Por lo antes expuesto debe existir un ambiente propicio para que el educando al interactuar con su medio y las estrategias aplicadas por el docente no encuentre dificultades y se produzca con eficacia el aprendizaje.

En este sentido, cobra importancia el juego como estrategia a través del cual se puede lograr la internalización de conocimientos. Ferrero (2003), cita que "los juegos de lápiz y papel tienen un carácter propedéutico en cuanto a que provocan en los alumnos un acercamiento a la geometría" (p.19).

Con estos juegos se pretende que el estudiante trabaje personalmente en problemas geométricos interesantes; no rutinarios, asequibles a su edad.

Los planteamientos hasta aquí expuestos motivan la idea de proponer a los juegos como estrategia metodológica en la enseñanza de la geometría para lograr un mejor entendimiento de la geometría.

\subsection{Marco teórico}

\subsubsection{Antecedentes}

A continuación se describirán algunos trabajos de investigación que han abordado el comportamiento de estudiantes y docentes frente al proceso de enseñanza y aprendizaje de la matemática.

Un valioso antecedente de esta investigación lo constituye el estudio realizado por Matos (1992). El objetivo de este trabajo fue comparar el rendimiento académico y la actitud hacia la matemática de dos grupos de estudiantes de séptimo grado de Educación Básica, sometidos a dos estrategias de enseñanza.

En 1997, Mata efectuó un trabajo de campo cuyo objetivo fue evaluar las actividades que generaban la aplicación de la matemática divertida en el quinto grado de Educación Básica.

Otro antecedente muy importante que tiene relación directa con nuestro objetivo general es el realizado por Cisneros (2004). El objetivo principal de este trabajo fue lograr que el aprendizaje de la matemática sea a partir de juegos donde se elaboren y resuelvan problemas.

Yépez (2004) presentó una investigación de campo, la cual consistió en diseñar y aplicar un taller del juego como estrategia de reforzamiento para facilitar el aprendizaje de las operaciones básicas y fracciones en niños y niñas de la II Etapa de Educación Básica.

Carrasco (2003), en su investigación apoyada en la modalidad de proyecto factible, presentó un diseño de un programa de geometría mediante el juego como estrategia pedagógica dirigida a los y las estudiantes de 6to grado de la II Etapa de Educación Básica.

Cabrera, Castro y Gutiérrez (2001) abordaron un estudio sobre los juegos como estrategia pedagógica para la enseñanza de las operaciones aritméticas básicas de 4to grado en tres escuelas del estado Anzoátegui, República Bolivariana de Venezuela.

Los trabajos citados anteriormente sirven de base para inferir la importancia del uso de adecuadas estrategias metodológicas en la enseñanza de la matemática para hacer más efectivo los procesos de enseñanza y de apren-

Los juegos como estrategia metodológica . Carmelo Bravo et al.

Derechos Reservados (C) 2012 Revista digital Matemática, Educación e Internet (www.tec-digital.itcr.ac.cr/revistamatematica/) 
dizaje.

\subsubsection{Bases teóricas}

Estrategias metodológicas para la enseñanza de las matemáticas. Se debe romper con esa enseñanza tradicional, en donde se transmite una gama de conocimiento que el estudiante debe recibir y posteriormente reflejar esos contenidos en un instrumento de evaluación. El docente debe inducir al estudiante que cada contenido matemático tiene una utilidad práctica en su quehacer diario y para qué le es útil. Es aquí donde el juego como estrategia de aprendizaje cobra vida dentro de la actividad diaria de clases.

El carócter lúdico del juego. Basados en las definiciones de juegos dadas por Piaget (1976), Ferrero (2003), Martínez (1996) y Huizinga (1968) nos permiten concluir que el juego es una actividad libre, que proporciona descanso, bajo ciertas reglas y tiene un fin en si misma, acompañado de sentimientos de tensión y alegría de suma importancia en la vida de todo ser humano, ya que la lúdica es inherente al hombre. Somos capaces de jugar indistintamente de nuestras edades.

Finalidad de los juegos. Parafraseando a Betancourt (2000), los juegos tienen una doble intención, ya que no se trata de lograr dos metas por separado, sino de manera conjunta es posible afirmar que estos juegos, si se emplean de manera adecuada se pueden convertir en instrumentos muy útiles para lograr una atmósfera eficiente en cuanto al desarrollo de los procesos psíquicos que conllevan a una mayor productividad grupal y que, a la vez, son satisfactorios para los participantes.

Hay que tomar en cuenta los aportes de Wallon (1942), Vygotsky (1978), Bruner (1987) y Piaget (1993) sobre la teoría del juego. Ellos coinciden que a nivel de la primera infancia, el juego cumple una tarea mediadora y actúan como un trampolín de un objeto a otro.

El juego en la educación. Para Ferrero (2003) el juego tiene un enorme valor educativo. Desde el punto de vista didáctico, los juegos favorecen que los estudiantes aprendan a desarrollar hábitos y actitudes positivas frente al trabajo individual y grupal, desde este punto de vista, el juego en el aula tiene un enorme valor como recurso didáctico convirtiéndose en un medio para facilitar la enseñanza.

Sin embargo, a pesar de esta afirmación en la mayoría de los casos los educadores evaden o no hacen uso del juego como estrategia metodológica que le permita mejorar o facilitar notablemente el aprendizaje del estudiante. Generalmente las tareas escolares sólo se limitan a obligar al discente a realizar un sin número de actividades que no son interesantes para él, estas actividades pueden ser: largas lecturas poco relevantes, resolución de problemas no acorde con la realidad, entre otros; lo cual es notorio la idea de dar un cambio en la metodología aplicada por los y las docentes, de tal manera que los y las estudiantes se involucren espontáneamente en las actividades a ser desarrolladas en el aula, donde el juego pudiera ser visto como una estrategia que rompa con ese tipo de enseñanza tradicional con la finalidad de despertar el interés, la curiosidad y el entusiasmo por aprender cada día más.

Con base a lo planteado anteriormente, se considera necesario emplear juegos atractivos y novedosos que estimulen al educando y a la vez propicien aprendizajes significativos.

El juego y la enseñanza de la matemática. Además de facilitar el aprendizaje de la matemática, el juego, debido a su carácter motivador, es uno de los recursos didácticos más interesantes que puede romper la aversión que los y las estudiantes tienen hacia esta asignatura.

Según CENAMEC (1998): la incorporación del juego de manera efectiva a los procesos de enseñanza y aprendizaje de la matemática, entre muchas posibilidades; puede ser utilizado: 
- Como motivador de un trabajo posterior (al jugar libremente con sólidos, el niño se da cuenta de las características de éstos).

- Para afianzar conceptos (juegos del valor de posición).

- Para reforzar las combinaciones de adición, sustracción, multiplicación y división.

- Como reforzador de los procesos de enseñanza y aprendizaje (uso de los juegos en la evaluación formativa.

- Representar una situación o problema de forma esquemática, es decir, construir un modelo de la situación, donde los alumnos y el docente logren precisar las reglas del juego, lo cual ayuda a los primeros a convertirse en actores y no en simples espectadores de la situación. Esto les permite arribar a conclusiones adecuadas acerca del modelo que hayan considerado.

El estudiante no juega para aprender matemática, pero por medio del juego desarrolla, de una manera intuitiva habilidades y destrezas matemáticas, que constituyen procesos cada vez más complejos, mediante el ejercicio fructífero de la imaginación.

El juego en la enseñanza de la geometría. Muchas de las limitaciones que los estudiantes manifiestan en la actualidad en el contexto educativo sobre su comprensión acerca de temas de geometría se deben al tipo de enseñanza que han recibido. Asimismo, el tipo de enseñanza que emplea el docente depende, en gran medida de las concepciones que él tiene sobre lo que es geometría, cómo se aprende, qué significa saber esta rama de las Matemáticas y para qué se enseña.

Es importante reflexionar sobre las razones para enseñar geometría. Si el docente tiene claro el porqué, estará en condiciones más acertadas acerca de su enseñanza, convirtiéndose en un docente capaz de implementar estrategias novedosas que desarrollan destrezas y habilidades en los estudiantes que le lleven a construir progresivamente el conocimiento geométrico, constituyendo los juegos didácticos una herramienta exitosa para la enseñanza de la geometría.

Con los juegos se pretende que el alumno trabaje personalmente en problemas geométricos interesantes, no rutinarios, adaptados a su edad y a su entorno; que realicen investigaciones sobre, entre otras cosas, propiedades geométricas; por otra parte la introducción de los juegos, en el aula de clases, en los que los aspectos geométricos estén presentes, sirven al menos, para compensar el tratamiento secundario que en los programas escolares de matemáticas se da a la geometría.

La geometría y los juegos tienen muchos rasgos en común en cuanto a la finalidad educativa, desarrolla en los estudiantes la capacidad de comprensión y representación del espacio. Los juegos enseñan a los estudiantes a desarrollar técnicas intelectuales, potenciar el pensamiento lógico, desarrollar hábitos de razonamiento y enseñan a pensar con espíritu crítico.

\subsection{Marco metodológico}

\subsubsection{Concepción metodológica de la investigación}

La presente investigación tuvo como propósito la presentación de los juegos como estrategia metodológica en la enseñanza de la geometría, para mejorar el rendimiento escolar de los estudiantes de séptimo grado de Educación Básica, de la Unidad Educativa. Para lograr este objetivo se aplicaron métodos y técnicas que permitieron exponer 
un orden metodológico, definir el tipo de investigación, la población, muestra y los instrumentos para recolectar los datos.

Tipo de investigación. El estudio que se llevó a cabo fue de tipo cuantitativo.

Diseño de investigación. Este estudio se ubicó en la investigación de campo, por cuanto la obtención de la información y recolección de los datos se hizo directamente de docentes y estudiantes investigados, lo que permitió el logro del objetivo.

Población y muestra. El conjunto de personas sobre quienes estuvo dirigida esta investigación lo conforman los estudiantes de las ocho (8) secciones de séptimo grado de matemáticas de la Unidad Educativa “Ricardo Márquez Moreno", ubicada en Santa Ana, estado Nueva Esparta, República Bolivariana de Venezuela , para un total de 200 estudiantes y ocho (08) docentes que dictan la asignatura en dichas secciones.

La muestra estuvo representada por 50 estudiantes integrantes de dos (2) secciones y fue una muestra intencionada, por considerarse que estos estudiantes tienen características similares a las de los demás estudiantes de dichas secciones.

Variable en estudio. En esta investigación se definió la variable "Los juegos como estrategia metodológica en la enseñanza de la geometría" en estudiantes de séptimo grado de Educación Básica de la Unidad Educativa, a través de estos, los estudiantes adquieren conocimientos dirigidos a la comprensión y resolución de problemas que se plantean, siguiendo una metodología de razonamiento

\subsubsection{Técnicas e instrumentos de recolección de datos}

En esta investigación las técnicas utilizadas fueron la observación directa y la encuesta las cuales se ejecutaron en función de los objetivos planteados.

El instrumento que se utilizó en base a la técnica señalada fueron dos cuestionarios que se aplicaron a toda la muestra, tanto de estudiantes como de docentes con el objeto de obtener los datos necesarios para la realización del presente estudio.

En la elaboración del cuestionario se tomó en cuenta los siguientes aspectos:

- Elaboración de un banco de ítems.

- Selección de ítems de acuerdo de acuerdo al objetivo planteado.

- Validación (validación del contenido).

Características del instrumento. El instrumento estuvo conformado por dos cuestionarios tipo Likert, con preguntas de respuestas cerradas, con cuatro (4) opciones, aplicado a docentes y estudiantes de séptimo grado de Educación Básica de la Unidad Educativa. Este instrumento estuvo conformado por 17 preguntas dirigidas a docentes y 9 a estudiantes. Ambos cuestionarios tenían en común evaluar las dimensiones e indicadores de la variable en estudio. Las dimensiones fueron tres y se describen: 
Dimensión: "Problemas de los estudiantes en el aprendizaje de la Geometría" y sus indicadores: aprendizaje memorístico, bajo rendimiento y participación y pasividad.

Dimensión: "Estrategias tradicionales en la enseñanza de la Geometría" y sus indicadores: regla y compás y exposición docente.

Dimensión: "Estrategias metodológicas actuales" y sus indicadores: conocimiento del docente de las estrategias actuales y juegos didácticos.

Validez del instrumento. Para la validación del cuestionario, se aplicó una de las formas más efectivas utilizadas en investigación: la validez del contenido y fue realizada por tres docentes Magister en Educación, los cuales avalaron el instrumento de recolección de datos.

\subsubsection{Técnicas de procesamiento y análisis de datos}

El procesamiento de datos de esta investigación se realizó en forma manual.

Luego de procesarse y tabularse de forma manual, se analizaron porcentualmente empleando para ello cuadros y gráficos, y se basó en la estadística descriptiva para su interpretación.

Finalmente atendiendo los indicadores de la variable en estudio, se analizaron cada uno de los ítems para poder conocer las evidencias con el problema planteado que es "los juegos como estrategia metodológica en la enseñanza de la geometría" en estudiantes de séptimo grado de la Unidad Educativa, y se determinó la relación entre el instrumento aplicado y los resultados obtenidos; a partir de los cuales se elaboraron las conclusiones y recomendaciones.

\subsection{Análisis de datos}

A continuación presentamos una idea de cómo se realizaron los análisis de los datos. Las dos primeras corresponden a la encuesta realizada a los docentes y las otras dos la realizada a los estudiantes.

Tabla 1.1 Frecuencias y porcentajes obtenidos en la Dimensión: Problemas de las y los estudiantes en el aprendizaje de la geometría. Indicador: aprendizaje memorístico.

\begin{tabular}{|l|l|l|l|l|l|l|l|l|l|l|}
\hline ALTERNATIVAS & \multicolumn{2}{l|}{ Siempre } & \multicolumn{2}{l|}{ Casi Siempre } & \multicolumn{2}{l|}{ Algunas Veces } & \multicolumn{2}{l|}{ Nunca } & \multicolumn{2}{l|}{ Total } \\
\hline ITEMS & $\mathrm{F}$ & $\%$ & $\mathrm{~F}$ & $\%$ & $\mathrm{~F}$ & $\%$ & $\mathrm{~F}$ & $\%$ & $\mathrm{~F}$ & $\%$ \\
\hline 1 & 6 & 75,00 & 1 & 12,50 & 1 & 12,50 & 0 & - & 8 & 100,00 \\
\hline 2 & 7 & 87,50 & 1 & 12,50 & 0 & - & 0 & - & 8 & 100,00 \\
\hline 3 & 8 & 100,00 & 0 & - & 0 & - & 0 & - & 8 & 100,00 \\
\hline Promedio & 7 & 88 & 1 & 8 & 0 & 4 & 0 & 0 & 8 & 100,00 \\
\hline
\end{tabular}

Ítem $\mathrm{N}^{\circ} 1$ : ¿En el desarrollo de las actividades diarias de clase, explica a los estudiantes ejercicios, esperando que este repita la operación?.

En el ítem $\mathrm{N}^{\circ} 1$, se observa que seis (06) de los docentes encuestados, $75 \%$, señalan que siempre esperan que los estudiantes repitan fielmente lo expuesto en clase, $12,5 \%$ respondió algunas veces y finalmente con el mismo porcentaje 12,5\% respondió algunas veces. Estos resultados evidencian un nivel alto $75 \%$ de aprendizaje memorístico 


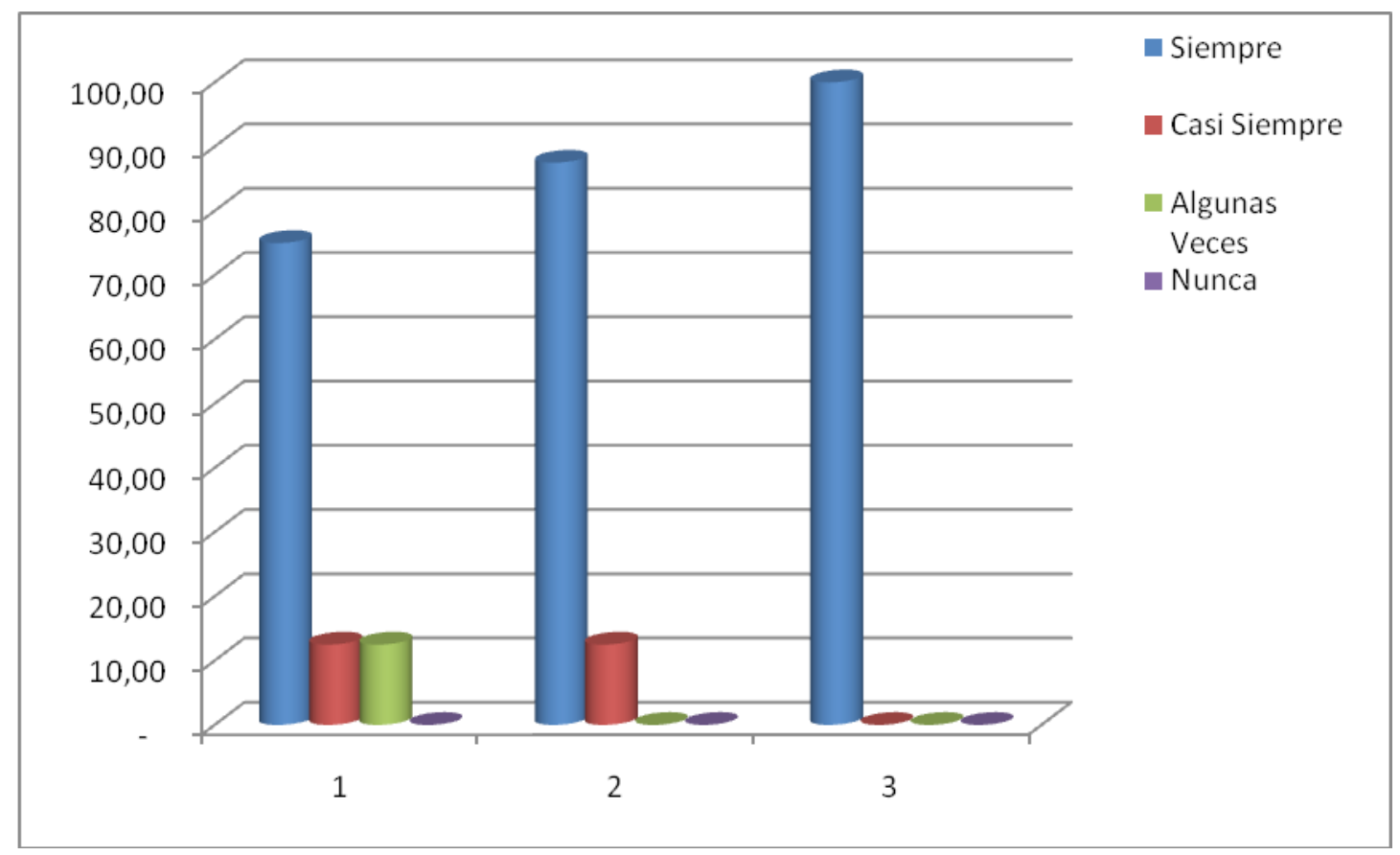

Figura 1.1 Porcentajes obtenidos en la dimensión problemas de los estudiantes en el aprendizaje de la geometría. Indicador: aprendizaje memorístico. Fuente: Elaboración propia.

Tabla 1.2 . Frecuencia y porcentajes obtenidos en la dimensión: estrategias actuales en la enseñanza de la geometría. Indicador: juegos didácticos.

\begin{tabular}{|l|l|l|l|l|l|l|l|l|l|l|}
\hline ALTERNATIVAS & \multicolumn{2}{l|}{ Siempre } & \multicolumn{2}{l|}{ Casi Siempre } & \multicolumn{2}{l|}{ Algunas Veces } & \multicolumn{2}{l|}{ Nunca } & \multicolumn{2}{l|}{ Total } \\
\hline ITEMS & F & $\%$ & F & $\%$ & F & $\%$ & F & $\%$ & F & $\%$ \\
\hline 14 & 0 & - & 0 & - & 1 & 12,50 & 7 & 87,50 & 8 & 100 \\
\hline 15 & 0 & - & 0 & - & 8 & 100,00 & 0 & - & 8 & 100 \\
\hline 16 & 8 & 100 & 0 & - & 0 & - & 0 & - & 8 & 100 \\
\hline 17 & 8 & 100 & 0 & - & 0 & - & 0 & - & 8 & 100 \\
\hline Promedio & 4 & 50 & 0,00 & 0,00 & 2,25 & 28,13 & 1,75 & 21,875 & 8 & 100 \\
\hline
\end{tabular}

impartido por los docentes.

Ítem $N^{\circ}$ 2: ¿Antes de formular preguntas a sus estudiantes, primero desarrolla toda la actividad de clase?

En este ítem se observa que siete (07) de los docentes de la muestra, es decir, el 87,5\% siempre desarrollan la clase antes de utilizar la estrategia de formular preguntas, mientras que solo un (01) docente que representa el 12,5\% respondió que casi siempre lo hace. A tal efecto se evidencia un nivel alto $87,5 \%$ de reforzamiento por parte del docente del aprendizaje memorístico.

Ítem $\mathrm{N}^{\circ}$ 3: ¿Es importante que los estudiantes repitan todo el contenido de su clase exactamente en las evaluaciones escritas?

En este ítem se refleja que la totalidad de los docentes (08), 100\% siempre esperan que sus estudiantes repitan los procedimientos exactamente, sin considerar procesos de análisis e interpretación de su parte, indicando esto un nivel muy alto $100 \%$ de aprendizaje memorístico. 


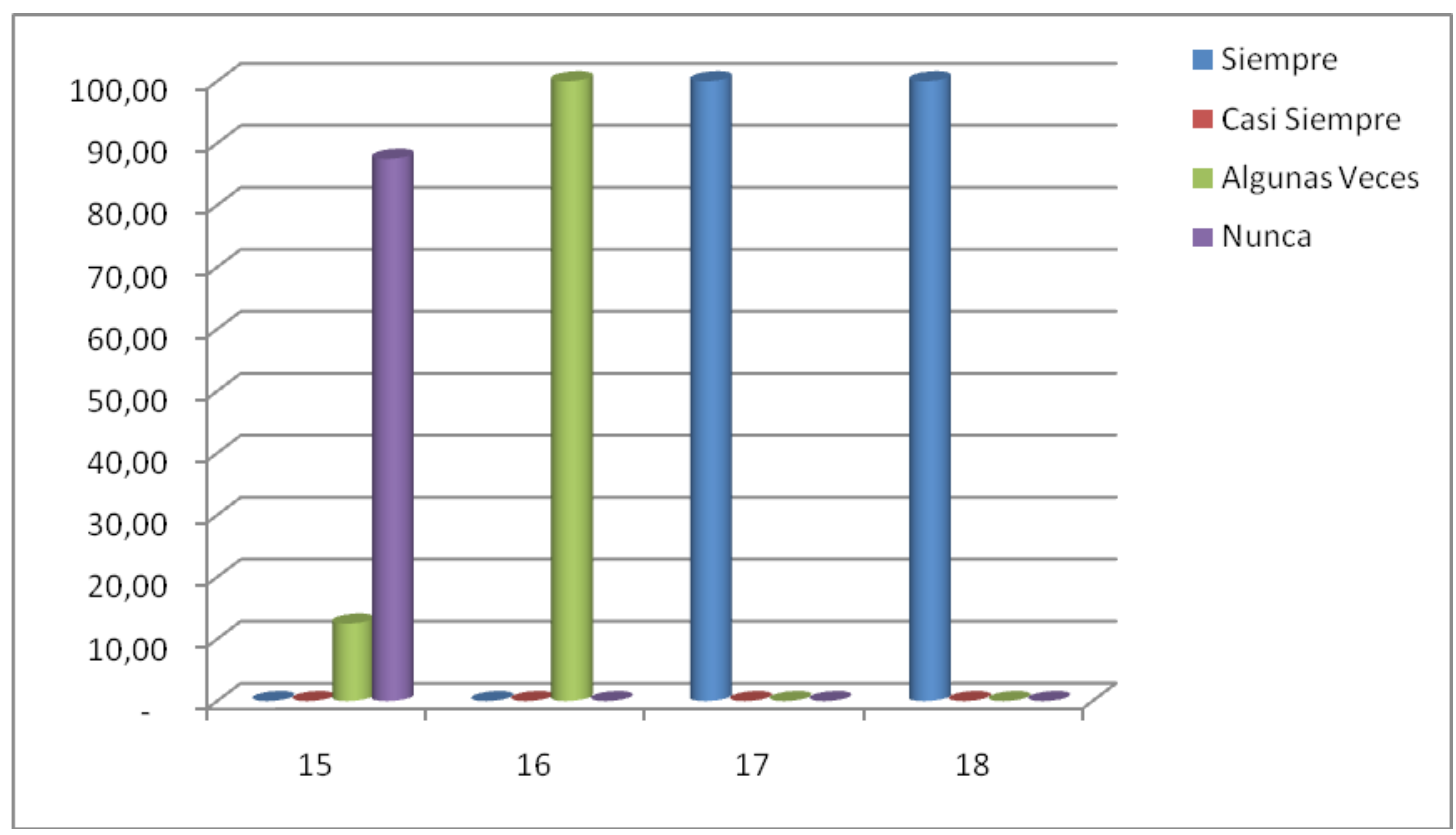

Figura 1.2 Porcentajes obtenidos en la dimensión: estrategias actuales. Indicador: juegos didácticos. Fuente: Elaboración propia

Ítem $N^{\circ} 14:$ ¿Utiliza juegos relacionados con la enseñanza de la geometría para que los estudiantes descubran por si mismos su aprendizaje?

De los resultados obtenidos se aprecia que un (01) docente que representa el 12,5\% utiliza algunas veces los juegos relacionados con la enseñanza de la geometría para que los estudiantes descubran por si mismos su aprendizaje, el resto de los docentes, el 87,5\% nunca lo utiliza, lo cual indica que los docentes carecen de las estrategias de juegos, imprescindibles para hacer de la enseñanza de la geometría una forma novedosa de impartir conocimientos y los estudiantes dejan de aprender con dicha estrategia.

Ítem $\mathrm{N}^{\circ} 15$ : ¿Es divertido que los estudiantes jueguen y aprendan en su actividad de clase?

Según los resultados obtenidos, se observa que la totalidad de los docentes, sólo utilizan la estrategia de juegos algunas veces, lo que demuestra la poca importancia que los docentes le dan a esta estrategia tan valiosa en el aprendizaje de los contenidos geométricos.

Ítem $\mathrm{N}^{\circ} 16:$ ¿El uso de los juegos didácticos como estrategia permite mejorar el rendimiento escolar?

Según la respuesta dada por los docentes, se aprecia en el gráfico que la totalidad de los docentes manifestaron que el uso de los juegos didácticos permite mejorar el rendimiento de los escolares, sin embargo lo utilizan muy pocas veces, debido quizás al desconocimiento de la estrategia o a la falta de tiempo para planificarla.

Ítem $\mathrm{N}^{\circ} 17:$ ¿Es importante la incorporación de los juegos didácticos como estrategia metodológica para la enseñanza de la geometría?

Aunque la totalidad de los docentes consideran que la incorporación de los juegos es importante para la enseñanza de la geometría, muy pocas veces la utilizan, demostrando con esto estar reacios al cambio, es decir, al uso de nuevas estrategias y al apego de las herramientas tradicionales, por lo que se hace necesario la sugerencia de talleres, lo 
cual es la propuesta que se hace en esta investigación.

Tabla 1.3 Frecuencias y porcentajes obtenidos en la dimensión: problemas de las y los estudiantes en el aprendizaje de la geometría. Indicador: aprendizaje memorístico.

\begin{tabular}{|l|l|l|l|l|l|l|l|l|l|l|}
\hline ALTERNATIVAS & Siempre & \multicolumn{2}{l|}{ Casi Siempre } & \multicolumn{2}{l|}{ Algunas Veces } & \multicolumn{2}{l|}{ Nunca } & \multicolumn{2}{l|}{ Total } \\
\hline ITEMS & $\mathrm{F}$ & $\%$ & $\mathrm{~F}$ & $\%$ & $\mathrm{~F}$ & $\%$ & $\mathrm{~F}$ & $\%$ & $\mathrm{~F}$ & $\%$ \\
\hline 1 & 38 & 76,00 & 8 & 16,00 & 2 & 4,00 & 2 & 4,00 & 50 & 100,00 \\
\hline 2 & 42 & 84,00 & 2 & 4,00 & 2 & 4,00 & 4 & 8,00 & 50 & 100,00 \\
\hline Promedio & 40 & 80 & 5 & 10 & 2 & 4 & 3 & 6 & 50 & 100,00 \\
\hline
\end{tabular}

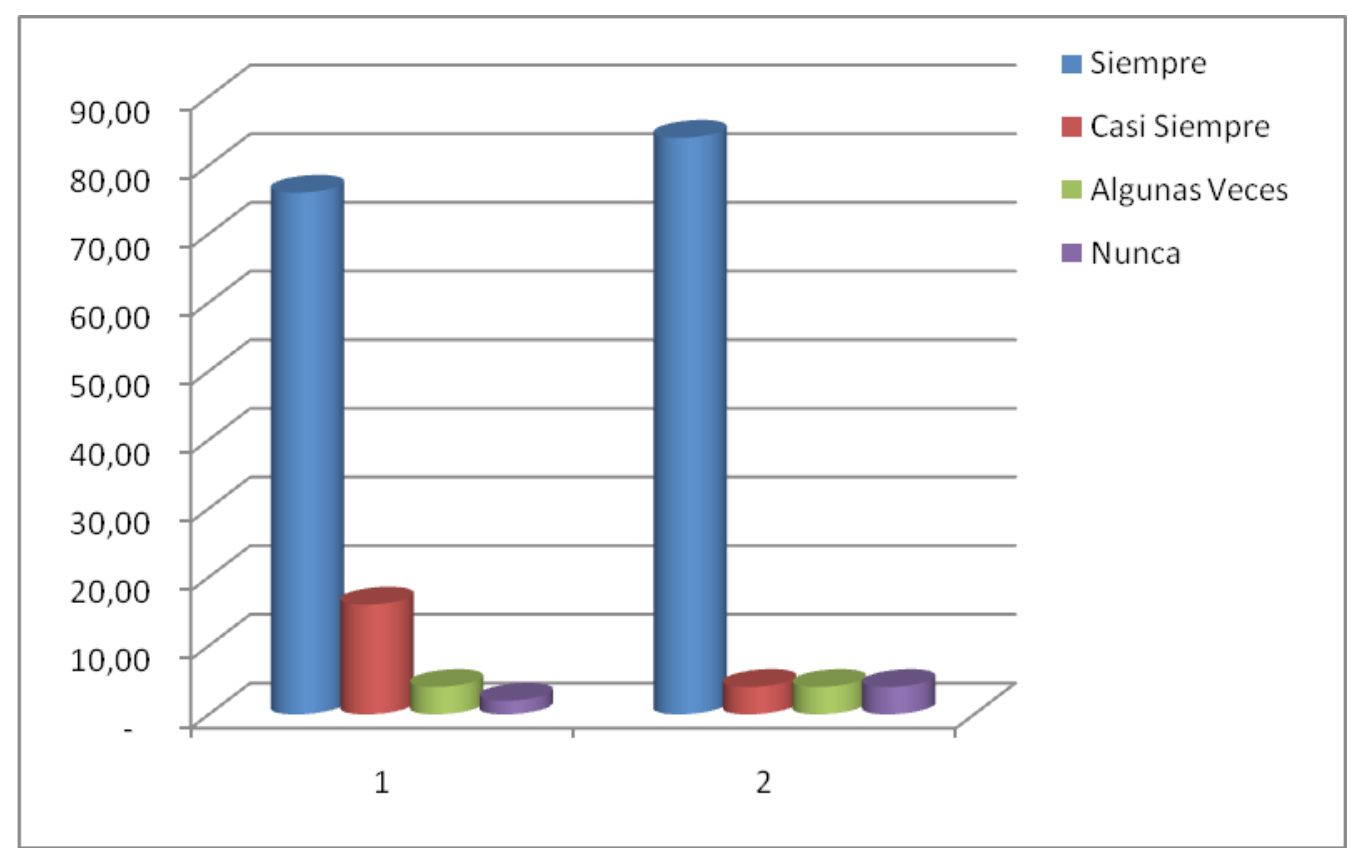

Figura 1.3 Porcentajes obtenidos en la dimensión problemas de las y los estudiantes en el aprendizaje de la geometría. Indicador: aprendizaje memorístico.

Ítem $\mathrm{N}^{\circ} 1$ : ¿En las actividades de la clase el docente, primero desarrolla la clase y luego formula las preguntas?

De acuerdo a los resultados obtenidos, el $76 \%$ de los estudiantes expresó que siempre las o los docentes primero desarrollan la clase y luego formula las preguntas, pero ocho (08) estudiantes, es decir el 16\% dicen que casi siempre, el $4 \%$ responde que algunas veces e igualmente el $4 \%$ de estudiantes responde que nunca lo hacen.

Estos resultados expuestos confirman la premisa de que la mayoría de los docentes permanecen como expositores dentro del aula de clase y los estudiantes reciben conocimientos que deben almacenarlos, para posteriormente responder en pruebas escritas, o en cualquier tipo de evaluación.

\section{Ítem $\mathrm{N}^{\circ}$ 2: ¿En las evaluaciones escritas debes responder con todo el contenido de las clases?}

En las respuestas a este ítem se refleja que la mayoría de los estudiantes, el $84 \%$ siempre deben responder con todo el contenido de la clase las evaluaciones escritas; aunque sólo dos (02) estudiantes, el $4 \%$ expresaron que casi siempre y algunas veces lo hacen, mientras que cuatro (04) estudiantes que representan el $8 \%$ nunca lo hacen. 
Tabla 1.4 . Frecuencia y porcentajes obtenidos en la dimensión: problemas de los estudiantes en la enseñanza de la geometría. Indicador: bajo rendimiento.

\begin{tabular}{|l|l|l|l|l|l|l|l|l|l|l|l|}
\hline ALTERNAIVAS & \multicolumn{2}{l|}{ Siempre } & \multicolumn{2}{l|}{ Casi Siempre } & \multicolumn{2}{l|}{ Algunas Veces } & \multicolumn{2}{l|}{ Nunca } & \multicolumn{2}{l|}{ Total } \\
\hline ITEMS & $\mathrm{F}$ & $\%$ & $\mathrm{~F}$ & $\%$ & $\mathrm{~F}$ & $\%$ & $\mathrm{~F}$ & $\%$ & $\mathrm{~F}$ & $\%$ \\
\hline 3 & 30 & 60,00 & 18 & 36,00 & 2 & 4,00 & 0 & - & 50 & 100,00 \\
\hline Promedio & 30 & 60 & 18 & 36 & 2 & 4 & 0 & - & 50 & 100,00 \\
\hline
\end{tabular}

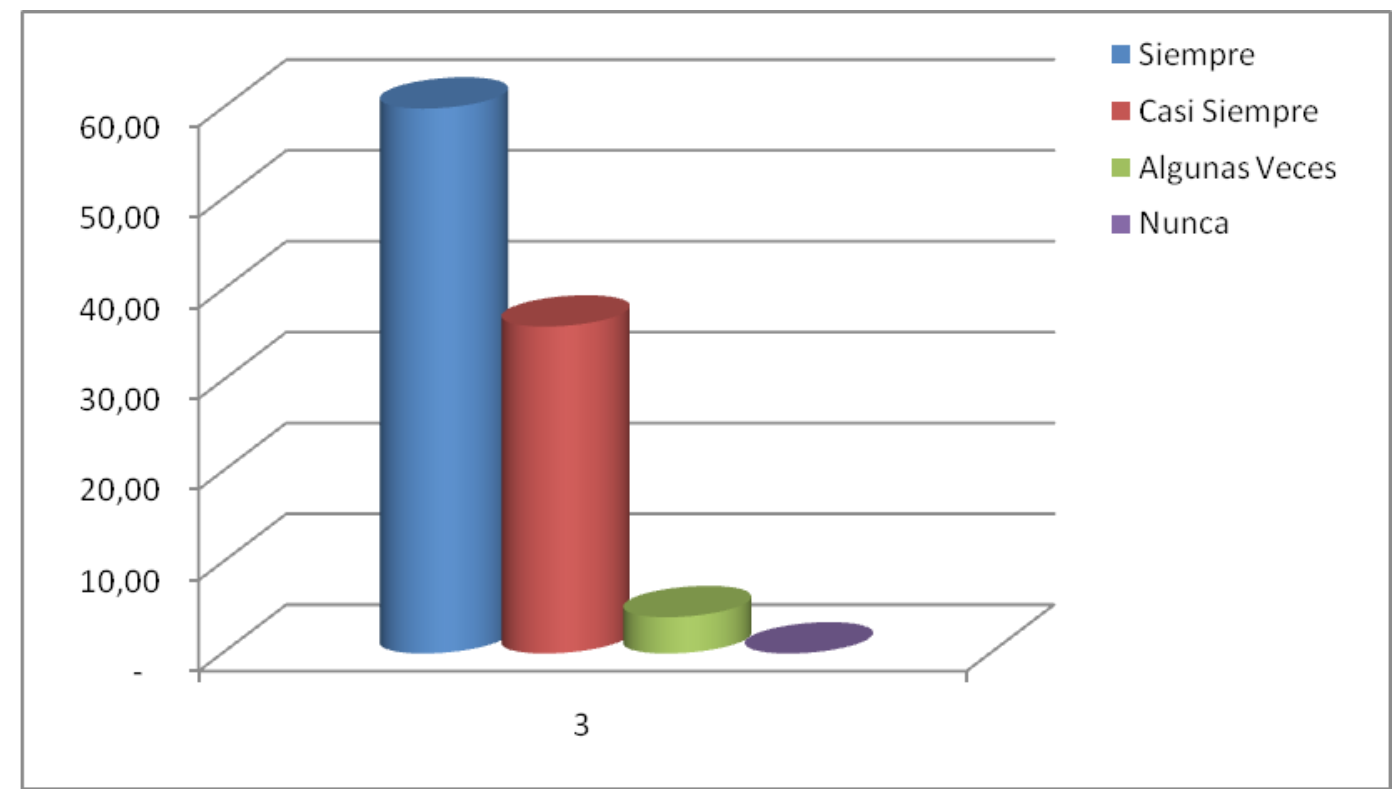

Figura 1.4 Porcentajes obtenidos en la dimensión: problemas de los estudiantes en la enseñanza de la geometría. Indicador: bajo rendimiento. Fuente: Elaboración propia

\section{Ítem Nº3: ¿Por lo general obtienes bajas calificaciones en matemáticas?}

De acuerdo con los resultados obtenidos, un número elevado de estudiantes, treinta (30), que representan el (60\%), siempre obtienen bajas notas en matemáticas, mientras que dieciocho (18) estudiantes, es decir, el (36\%) casi siempre obtiene bajas calificaciones, y sólo dos (02) estudiantes, el $4 \%$ algunas veces. Esto demuestra que el rendimiento de la mayoría de los estudiantes en matemáticas de séptimo grado es muy bajo.

\subsection{Conclusiones}

Es necesario transformar la realidad de la educación matemática en particular de la geometría en séptimo grado de Educación Básica, puesto que esta disciplina es fundamental en la formación integral de los estudiantes. Por lo que se hace necesario fortalecer la capacidad docente a fin de posibilitar una pedagogía de enseñanza.

Se observó que los docentes hacen uso de estrategias tradicionales, como la exposición en la enseñanza de la geometría, y el uso de la pizarra para escribir definiciones y fórmulas, es decir no promueven la participación activa de los estudiantes, como son las estrategias dinámicas, lo que trae como consecuencia la pasividad, la falta de mo-

Los juegos como estrategia metodológica . Carmelo Bravo et al.

Derechos Reservados (C) 2012 Revista digital Matemática, Educación e Internet (www.tec-digital.itcr.ac.cr/revistamatematica/) 
tivación y el bajo rendimiento.

Los docentes utilizan muy pocas veces los juegos didácticos como estrategia para la enseñanza de la geometría, por lo que deben hacer uso de ellas como actividad motivadora y significativa que despierte las potencialidades de las y los estudiantes.

Los estudiantes necesitan motivación e integración hacia el tópico matemático (geometría), mediante una estrategia motivadora y agradable como la de los juegos didácticos, que les permita lograr el aprendizaje por su propio esfuerzo, incentivando la autoestima, la perseverancia, la motivación al logro, los cuales son valores esenciales en la formación del estudiante.

\subsection{Recomendaciones}

En las instituciones educativas se deben realizar talleres donde se tome en cuenta el juego como estrategia para la enseñanza de la geometría para facilitar el desarrollo y anclaje de los contenidos de este tópico, en un ambiente armonioso, en donde la participación del estudiante sea espontánea, que ellos puedan dejar un producto final, el cual sea logrado con mucha constancia, que se sientan orgullosos por el trabajo emprendido.

Se propone la realización de "Talleres mediante el juego" como estrategias metodológicas en la enseñanza de la geometría para que los docentes de matemáticas puedan aplicarlos a los estudiantes de séptimo grado de Educación Básica.

Los docentes en servicio deberían organizarse con el fin de discutir y analizar la problemática actual de la Educación Básica con el fin de hallar posibles cambios.

\section{Bibliografía}

[1] Betancourt, J. Atmósferas creativas, juega, piensa y crea. Manual Moderno. México. 2000.

[2] Bruner, J. Concepción de la infancia: Freud, Piaget y Vigotsky, en Linaza, J.L (compilador) Acción, pensamiento y lenguaje. Alianza Editorial. Madrid. España. 1987.

[3] Cabrera, M., Castro, E. y Gutiérrez, B. Uso de los juegos como estrategia pedagógica de las operaciones aritméticas básicas de 4to grado en tres escuelas del área Barcelona Naricual. Tesis. Anzoátegui. Venezuela. 2001.

[4] Carrasco, M. Diseño de un programa de geometría mediante el juego como estrategia pedagógica. Barquisimeto. UNA. 2003.

[5] CENAMEC. Carpeta de Matemática para docentes de Educación Básica. Volumen uno. Segunda edición. Caracas.1998.

[6] CENAMEC. Encuentro compartiendo experiencias en las áreas de matemáticas y ciencias de la naturaleza. Ministerio de Educación. Caracas: autor. 2001.

[7] Cisneros, I. El juego didáctico en el aprendizaje de las matemáticas en la resolución de problemas. Tesis en línea. Universidad La Salle Benavente, Puebla. México. en http: // ficha obra. Html? Ref. =2019. Consultada el 19 de abril de 2007

[8] Currículo Básico Nacional. Caracas: ME. 1987

[9] Ferrero, L. El juego y la matemática. Madrid. La Muralla. S.A. 2003.

[10] Huizinga, J. El concepto de juego y sus expresiones en el lenguaje. Buenos Aires. Homo Ludens. 1968.

[11] Martínez, C. El Juego y el Desarrollo Infantil. Barcelona. Otaedro. 1996.

Los juegos como estrategia metodológica. Carmelo Bravo et al.

Derechos Reservados (C) 2012 Revista digital Matemática, Educación e Internet (www.tec-digital.itcr.ac.cr/revistamatematica/) 
[12] Mata, M. Propuesta para aplicar estrategias cognoscitiva en el proceso de enseñanza y aprendizaje en la matemática para la I etapa de educación básica. (Trabajo monográfico). La Asunción. UPEL. I.M.P.M. 1997.

[13] Matos, L. Efectos cognoscitivos de dos estrategias institucionales fundamentales. En revista de investigación y postgrado. Caracas, UPEL. IMPM. Volumen 7., p.64 y 76. 1992.

[14] Modelo Normativo de Educación Básica. Caracas: M.E. 1987.

[15] Piaget, J. La representación del mundo en el niño. Ediciones Morata. Madrid. España. 1993.

[16] Piaget, J. La función semiótica o simbólica en la psicología del niño. Ediciones Morata. España. 1976.

[17] Sistema Nacional de Medición. Ministerio de Educación.. (SINEA). Informe para el docente. Caracas: Autor. 1998

[18] Vygotsky, L. Desarrollo de los procesos psicológicos superiores. Editorial Crítica, Grijalbo. Barcelona. España. 1978.

[19] Wallon, H. El juego, en la evolución psicológica del niño. Editorial Psique. Buenos Aires. Argentina. 1942.

[20] Yépez, M. Diseño y aplicación de un taller del juego como estrategia de reforzamiento en la enseñanza de las operaciones básicas y fracciones en niños de la II Etapa de Educación Básica. Caracas. UNA. 2004.

[21] Zambrano, A. Conocimiento, saber y pensamiento: una aproximación a la didáctica de las matemáticas. EquisAngulo, No.1, 1-6. 2005.

Los juegos como estrategia metodológica . Carmelo Bravo et al.

Derechos Reservados @ 2012 Revista digital Matemática, Educación e Internet (www.tec-digital.itcr.ac.cr/revistamatematica/) 